\title{
The selective treatment of clinical mastitis based on on-farm culture results: II. Effects on lactation performance, including clinical mastitis recurrence, somatic cell count, milk production, and cow survival
}

\author{
A. Lago, ${ }^{* 1}$ S. M. Godden, ${ }^{*}$ R. Bey, ${ }^{*}$ P. L. Ruegg, $†$ and K. Leslieł \\ *Department of Veterinary Population Medicine, College of Veterinary Medicine, University of Minnesota, Saint Paul 55108 \\ †Department of Dairy Science, College of Agriculture and Life Sciences, University of Wisconsin, Madison 53706 \\ ¥Department of Population Medicine, Ontario Veterinary College, University of Guelph, Ontario, Canada N1G 2W1
}

\begin{abstract}
The objective of this multi-state, multi-herd clinical trial was to report on the efficacy of using an on-farm culture system to guide strategic treatment decisions in cows with clinical mastitis. The study was conducted in 8 commercial dairy farms ranging in size from 144 to 1,795 cows from Minnesota, Wisconsin, and Ontario, Canada. A total of 422 cows affected with mild or moderate clinical mastitis in 449 quarters were randomly assigned to either (1) a positive-control treatment program or (2) an on-farm culture-based treatment program. Quarter cases assigned to the positive-control group received immediate on-label intramammary treatment with cephapirin sodium. Quarters assigned to the culture-based treatment program were not treated until the results of on-farm culture were determined after 18 to $24 \mathrm{~h}$ of incubation. Quarters in the culture-based treatment program that had gram-positive growth or a mixed infection were treated according to label instruction using intramammary cephapirin sodium. Quarters assigned to the culture-based treatment program that had gram-negative or no-growth did not receive intramammary therapy. It was already reported in a companion paper that the selective treatment of clinical mastitis based on on-farm culture results decreases antibiotic use by half and tends to decrease milk withholding time without affecting short-term clinical and bacteriological outcomes. The present article reports on long-term outcomes of the aforementioned study. No statistically significant differences existed between cases assigned to the positive-control program and cases assigned to the culture-based treatment program in risk and days for recurrence of clinical mastitis in the same quarter ( $35 \%$ and $78 \mathrm{~d}$ vs. $43 \%$ and 82 d), linear somatic cell count (4.2 vs. 4.4), daily milk production (30.0 vs. $30.7 \mathrm{~kg})$, and risk and days for culling or death
\end{abstract}

Received November 28, 2010.

Accepted May 23, 2011.

${ }^{1}$ Corresponding author: alfonso.lago@gmail.com events (28\% and $160 \mathrm{~d}$ vs. $32 \%$ and $137 \mathrm{~d}$ ) for the rest of the lactation after enrollment of the clinical mastitis case. In summary, the selective treatment of clinical mastitis based on on-farm culture resulted in no differences in long-term outcomes, such as recurrence of clinical mastitis in the same quarter, somatic cell count, milk production, and cow survival for the rest of the lactation after clinical mastitis.

Key words: clinical mastitis, selective treatment, onfarm culture, bi-plate

\section{INTRODUCTION}

Mastitis remains the most costly infectious disease, and the most frequent reason for antibacterial use on commercial dairy farms (Sundlof et al., 1995; Mitchell et al., 1998; Pol and Ruegg, 2007). As such, research should continue on the development and validation of new management tools that will help decrease the health and economic impact of this disease, while at the same time promoting the judicious and strategic use of antimicrobials on dairy farms.

It has been suggested that clinical mastitis cases that yield no bacterial growth and cases from which gramnegative bacteria are isolated may not benefit from intramammary antimicrobial therapy (Erskine et al., 1992; Pyörälä et al., 1994; Roberson, 2003). Conversely, intramammary antibiotic therapy is routinely recommended for infections caused by gram-positive organisms. Consequently, clinical mastitis treatment decisions should be based on culture results. Adoption of rapid on-farm milk culture systems, such as the Minnesota Easy Culture System (University of Minnesota, Saint Paul, MN), could allow veterinarians and producers to make strategic treatment decisions for clinical mastitis cases, based on knowing the pathogen involved.

A companion paper has described how the selective treatment of clinical mastitis based on on-farm culture results decreases antibiotic use by half and tends to decrease milk withholding time without affecting shortterm clinical and bacteriological cure outcomes (Lago 
et al., 2011). However, the long-term performance of the quarter and cow also represents an important component of a clinical mastitis treatment program. For example, in previous clinical mastitis field trials comparing antibacterial and no antibacterial clinical mastitis treatments, the risk for recurrence of clinical mastitis did differ for mild clinical mastitis cases where streptococci were isolated (Van Eenennaam et al., 1995). In addition, quarter milk SCC has been shown to be greater after clinical mastitis (Sheldrake et al., 1983; Schepers et al., 1997; De Haas et al., 2002). Likewise, clinical mastitis results in milk production losses for the rest of the lactation (Houben et al., 1993; Rajala-Schultz et al., 1999; Wilson et al., 2004). In conventional US production systems, milk production losses associated with clinical mastitis has been estimated to be over $500 \mathrm{~kg}$ over the entire lactation (Wilson et al., 2004). However, because mature cows that suffer clinical mastitis are higher producers before the clinical mastitis event than their herdmates, the milk production losses over the entire lactation exceeds $1,000 \mathrm{~kg}$ over the entire lactation. Similarly, several studies have reported that clinical mastitis significantly increases the risk of culling (Dohoo and Martin, 1984; Erb et al., 1985; Milian-Suazo et al., 1989; Gröhn et al., 1997; Rajala-Schultz et al., 1999; Gröhn et al., 2005). The culling hazard rate for clinical mastitis cows was estimated to be more than twice that of non-clinical mastitis cows (Gröhn et al., 2005).

The objective of this study was to investigate the efficacy of using an on-farm culture system to guide strategic treatment decisions in cows with mild and moderate clinical mastitis, in regard to long-term outcomes in the same lactation including (1) risk and days to a recurrence of a clinical mastitis event in the same quarter, (2) cow survival post-enrollment (culling and death events), (3) SCC, and (4) milk production.

\section{MATERIALS AND METHODS}

\section{Study Design}

A randomized controlled field trial was conducted between June 2005 and April 2007 in a convenience sample of 8 commercial dairy farms from the North American Great Lakes region. Herd size, housing systems, milk production, and SCC are described elsewhere (Lago et al., 2011).

\section{Case Definition}

Clinical mastitis was diagnosed if milk from one or more glands was abnormal in color, viscosity, or consistency, with or without accompanying heat, pain, red- ness, or swelling of the quarter, or generalized illness. All lactating cows in the herd were eligible for enrollment at the time of clinical mastitis occurrence when only the milk was abnormal (mild or grade 1 clinical mastitis) or when the milk and the quarter were affected (moderate or grade 2 clinical mastitis). Cows exhibiting severe or grade 3 clinical mastitis (depression, anorexia, dehydration, fever) or any cow with fewer than 3 functional quarters were ineligible for enrollment.

\section{Enrollment Process}

Cows with clinical mastitis were detected in the milking parlor by the milkers upon appreciation of clinical signs of mastitis (e.g., visible abnormal milk or quarter). If the cow met the designated inclusion criteria for enrollment, herd personnel aseptically collected a single milk sample from the affected quarter. For a first clinical mastitis episode (cow not previously enrolled into the study), eligible cows for enrollment were randomly assigned following a simple randomization schedule to either the positive-control group or culture-based treatment group by opening a sealed opaque pre-identified envelope following a sequential order. If more than 1 quarter was affected, all affected quarters were assigned to the same treatment group. For a second (or greater) clinical mastitis episode in the same cow (i.e., cow had been previously enrolled) in the same or in a different quarter, the quarter was assigned to the same treatment group as was previously assigned.

\section{Treatment Groups}

Positive-Control Group. Immediately after enrollment the quarter milk sample that had been aseptically collected was frozen on-farm at $-20^{\circ} \mathrm{C}$ and the affected quarter(s) were infused with 1 syringe (200 mg) of cephapirin sodium (Cefa-Lak; Fort Dodge Animal Health Inc., Fort Dodge, IA). The treatment was repeated once, $12 \mathrm{~h}$ after the first treatment, according to label directions. A milk-withdrawal period of $96 \mathrm{~h}$ and a slaughter-withdrawal period of $4 \mathrm{~d}$ were followed after the last treatment.

Culture-Based Treatment Group. The aseptically collected milk sample(s) from the affected quarter(s) was first cultured on-farm using the Minnesota Easy Culture System (University of Minnesota, St. Paul, MN). This on-farm culture system consists of a bi-plate, which is a Petri dish with 2 different types of agar: MacConkey agar on one half that selectively grows gram-negative organisms and Factor agar, similar to KLMB agar (Beatty et al., 1985), on the other half of the plate that selectively grows gram-positive organisms while inhibiting the growth of gram-negative 
bacteria with antibiotics (University of Minnesota, St. Paul, MN). A sterile cotton swab was dipped into the milk sample and then plated onto the Factor media half of the bi-plate, redipped into the milk, and then applied to the MacConkey media half of the bi-plate. The plate was placed in an on-farm incubator and incubated at approximately $37^{\circ} \mathrm{C}$ for $24 \mathrm{~h}$. The quarter milk sample that had been collected was then frozen on-farm at $-20^{\circ} \mathrm{C}$. The next day, the plate was read and interpreted according to guidelines provided for the Minnesota Easy Culture System II user's manual (University of Minnesota Laboratory for Udder Health, 2004). If bacteria did not grow, the plate was returned to the incubator and re-read approximately $24 \mathrm{~h}$ later. Final results for each sample plate were recorded as (1) gram-positive, when bacteria grew only in the Factor agar media of the bi-plate; (2) gram-negative, when bacteria grew only in the MacConkey agar media of the bi-plate; (3) no-growth, when bacteria did not grow in either media; or (4) mixed infection when bacteria grew in both media. The decision about initiation of intramammary antibiotic therapy the day after enrollment of the clinical mastitis case was based on the on-farm culture results. Quarters from which grampositive bacteria were isolated or had a mixed infection received the same intramammary antibiotic treatment following the same procedures than cases assigned to positive-control treatment. If the on-farm culture result was gram-negative or no-growth, then the quarter did not receive intramammary therapy. Study technicians visited the study herds once per week and observed herd personnel performing on-farm culture procedures, recorded incubator temperature and evaluated the incubator humidity conditions, and revised the bi-plate colony growth readings done by farm personnel during the previous week (used bi-plates were kept in the refrigerator by farm personnel until technicians visited the farm once per week).

Long-term outcomes for the next $365 \mathrm{~d}$ or the end of the current lactation, whichever came first, recorded by farm personnel or captured through DHIA records, included whether or not the quarter experienced a relapse of clinical mastitis, milk production, SCC, and sale or death.

\section{Laboratory Bacteriological Culture}

Aerobic culture methodologies for frozen milk samples (enrollment d 0, 14, and 21) collected on farms were standardized among labs at all 3 participating sites and performed in accordance with the National Mastitis Council guidelines (NMC, 1999). Detailed laboratory procedures were described elsewhere (Lago et al., 2011).

\section{Data Analysis-Definition of Outcome Variables}

Recurrence of Clinical Mastitis. A recurrence was defined as detection of a new clinical mastitis case in the same quarter at least $14 \mathrm{~d}$ after enrollment of the previous case of clinical mastitis. Cow identification, affected quarter(s), and date of recurrent clinical mastitis cases were retrieved from dairy farm management or study records. Cows were followed until a new clinical mastitis event happened, the end of the current lactation or 12 mo after enrollment (whichever came first).

Removal from the Herd. For all cows in the study, the removal date (culling/death) was retrieved from the on-farm record-keeping system (DairyComp305; Valley Agricultural Software, Tulare, CA). Cows were followed until culling/death, the end of the current lactation, or up to 12 mo after enrollment (whichever came first).

SCC and Milk Production. Monthly DHIA SCC and milk production records from individual cows were retrieved for the entire lactation from the onfarm record keeping system (DairyComp305; Valley Agricultural Software, Tulare, CA). The test records used in this analysis were those that tracked until the end of the current lactation or 12 mo after enrollment (whichever came first). Milk SCC were transformed to normalize the data to linear SCC (LSCC) using the linear SCC formula: $\mathrm{LSCC}=[\operatorname{Ln}(\mathrm{SCC} / 100) / \operatorname{Ln}(2)]+$ 3 (Ali and Shook, 1980).

\section{Statistical Analysis_Models and Modeling Strategy}

Database summaries and plots were used for exploratory data analysis. Basic diagnostics techniques were used to evaluate normality, independence, homoscedasticity, collinearity, and linearity of variables.

General Linear Mixed Models for Continuous Outcome Variables. Continuous outcome variables, such as milk yield and LSCC, were modeled as a function of explanatory variables using linear multivariable regression. A multilevel general linear mixed model was constructed with milk yield/LSCC as a continuous, normally distributed response variable. The model was specified with random variation allowed in 3 hierarchical levels: repeated measure of milk yield/LSCC within cow, variation among cows within the herd, and variation among herds. This was accomplished with the MIXED procedure of SAS version 9.1 (SAS Institute, 2003) by specifying a correlation structure among the repeated measurements of the same cow [repeated test/ subject $=$ cow type $=\operatorname{ar}(1) \mathrm{r}$ rcorr] and including a random statement to account for clustering of cows within herds (random herd/subject $=$ cow). The general linear mixed models were specified as $Y_{i j}=\alpha+\boldsymbol{\beta}_{1 \mathbf{i}}^{\prime} \mathbf{X}_{1 \mathbf{i}}+v_{i}+u_{j}$, where the subscripts $i$ and $j$ 
denote the $i$ th cow and the $j$ th herd; $Y_{i j}=$ the fitted prediction for the $i$ th cow and the $j$ th herd; $\alpha=$ regression intercept; $\mathbf{X}_{1 \mathrm{i}}=$ vector of covariates associated with cow $i ; \boldsymbol{\beta}_{1 \mathrm{i}}^{\prime}=$ vector of coefficients for $\mathbf{X}_{1 \mathrm{i}} ; v_{i}=$ residual variation between cows; and $u_{j}=$ random effect reflecting clustering of cows within herds. To select the most appropriate covariance structure, we started with a full model with all confounding covariates already taken into account. Summary statistics and exploratory data analysis plots to explore the covariance structure were created, and one model was fitted for each covariance structure. The correlation structures that were evaluated include simple (no correlation), compound symmetry, banded diagonal, autoregressive, and unstructured (estimating a correlation for each separate correlation). The different correlation structures were evaluated using goodness-of-fit measures. The goodness-of-fit measures included $-2 *$ log-likelihood $(-2 \mathrm{LL})$, Akaike's information criterion, and Bayesian information criterion.

Covariates such as cow parity, DIM at clinical mastitis event, previous occurrence of a clinical mastitis case in the same quarter in the present lactation, number of quarters affected, case severity, and etiology of infection were included in the model if it was a potential confounding variable. Other covariates, such as LSCC in the test before the clinical mastitis event, were evaluated only when the estimated outcome was LSCC, and milk production in the test before the clinical mastitis event when the estimated outcome was milk production. To determine if a covariate confounded the treatment effect on the outcome (milk yield or LSCC), the crude estimate of treatment group (positive control- vs. culture-based treatment) was compared with the adjusted estimate for that third variable. It was concluded that the variable confounded the association between treatment group and outcome variable if the ratio between the difference of the crude estimate and the adjusted estimate versus the crude estimate was greater than $10 \%$. Each variable was examined for potential confounding 1 at a time by regression. Once the confounder variables were identified, the next step was to place all confounders into a full model with 2-way interaction terms between treatment and the confounder. To simplify the model, each nonsignificant interaction term was removed 1 at a time using a backward stepwise approach, starting with the least significant interaction term, and running the model again until no nonsignificant interaction terms existed in the model. Next, with nonsignificant interaction terms removed from the model, it was determined whether covariates existed in the model that were not in an interaction term that might be a confounder. The least significant term was removed and it was evaluated if this affected the treatment effect estimate, with the goal being to assess whether the variable confounded the treatmentoutcome relationship. If the variable was an important confounder, it was returned to the model and other variables were assessed 1 at a time to see if they were confounders. The treatment variable was forced in the model. Once all nonsignificant interaction terms were removed, as well as covariates that did not confound the exposure-outcome relationship, this was the final model. The MIXED PROC constructs an approximate t-test and significance was declared at $P<0.05$.

Time-to-Event Models. Binary responses with a time-to-event component, such as quarter risk and days to a clinical mastitis recurrence or risk and days to removal from herd (culling/death), were modeled using survival analysis. The Cox proportional hazards regression method was used to test the logistic analysis explanatory variables (see previously described covariates) simultaneously for their association with time until event (PROC TPHREG). The Cox model assumes a proportional hazard constant over time. This assumption was examined by the log-cumulative hazard plot to check if the lines for both treatment groups were parallel and tested by modeling an interaction between cohort and the follow-up time. The clustering of cows within herd (recurrence of clinical mastitis and removal from the herd outcomes) and quarters within cows (only for the recurrence of clinical mastitis outcome) was considered by specifying the Covsandwich (aggregate) option in the procedure statement and then specifying herd (id = herd) or herd and cow as the level of aggregate (id = herd cow). The Covsandwich option requests a robust sandwich estimate for the covariance matrix which results in a robust standard error for the parameter estimates. The survival function was modeled as $\lambda_{i}=\lambda_{0} * \exp \left(\beta x^{\prime}\right)$, where $\lambda_{i}=$ hazard function (risk of the event of interest in cow $i$ for removal from herd (or quarter $i$ for recurrence of clinical mastitis) at time $t$, where $t$ is the follow-up time; $\lambda_{0}=$ baseline hazard; $\beta x^{\prime}=$ linear predictor containing a vector of covariates $x$, with regression coefficients $\beta$.

For the clinical mastitis recurrence model, cows and quarters were censored if the cow was removed from herd, or if she survived in the herd to the end of the current lactation or at the end of a 12-mo follow-up period (whichever occurred first), without having experienced a recurrent clinical mastitis event. For the removal from herd model, cows were censored if they survived in the herd to the end of the current lactation or at the end of a 12-mo follow-up period. The assumption of independent censoring between both treatment groups was assessed by comparing the proportion of 
censored cows or quarters between both treatment groups. In addition, a sensitivity analysis looking at situations of complete positive correlation (every cow or quarter censored experienced the event of interest) or negative correlation (censored cows or quarters did not experienced the event of interest) between censoring and the event of interest was done. If the violation of this assumption did not dramatically alter the treatment effect estimate $(<10 \%)$, it was concluded that censoring did not introduce bias.

\section{RESULTS}

\section{Descriptive Data}

A total of 422 cows affected with clinical mastitis in 449 quarters were enrolled in the study. Of those, 214 cows with 229 affected quarters were assigned to positive-control treatment, and 208 cows with 220 affected quarters were assigned to culture-based treatment. Cowand quarter-level descriptors and etiology of infection at enrollment for both study groups were described previously in a companion paper (Lago et al., 2011).

\section{Clinical Mastitis Treatment Program Effects}

Recurrence of Clinical Mastitis. No significant differences existed in the risk and days to recurrence of a clinical mastitis event hazard ratio between treatment programs [positive-control hazard ratio (95\% CI) $=1.2(0.9,1.6) ; P=0.201$; Table 1 and Figure 1]. This risk was numerically higher in cases assigned to positive-control treatment in 3 herds, the same in 1 herd, and lower in 3 of the 7 herds where risk and days to recurrence of a clinical mastitis event data was available. Clinical mastitis recurrence data was not available for 1 herd because of misplacement of follow-up clinical mastitis case records. Covariates that remained in the model because of confounding of the treatment program effect on the recurrence of clinical mastitis included number of quarters affected and the occurrence of a previous case of clinical mastitis in the same quarter in the present lactation.

The risk and average days after enrollment to recurrence of a clinical mastitis event in the same quarter was $35 \%$ and $78 \mathrm{~d}$ and $43 \%$ and $82 \mathrm{~d}$ for cases assigned to positive-control and to culture-based treatment, respectively. The overall risk and average days to recurrence of a clinical mastitis event for no growth, gram-negative and gram-positive cases were $41 \%$ and $81 \mathrm{~d}, 45 \%$ and $79 \mathrm{~d}$, and $32 \%$ and $76 \mathrm{~d}$, respectively (Table 2).

Removal from the Herd. No significant difference existed in the removal from the herd hazard ratio between treatment programs [positive-control hazard ratio $(95 \% \mathrm{CI})=1.1(0.7,1.6) ; P=0.560 ;$ Table 1 and Figure 2]. This risk was numerically higher in cases assigned to the positive-control treatment in 4 herds and lower in 4 of the 8 herds enrolled in the study. Covariates that remained in the model because of confounding the treatment program effect on the risk and days to removal included parity of the cow, DIM at enrollment, and etiology of infection.

The risk and average days after enrollment to a culling or death event was $28 \%$ and $160 \mathrm{~d}$ and $32 \%$ and 137 $\mathrm{d}$ for cases assigned to positive-control and to culturebased treatment, respectively. The overall risk and average days to a culling/death event for no growth, gram-negative, and gram-positive cases were $24 \%$ and $155 \mathrm{~d}, 28 \%$ and $127 \mathrm{~d}$, and $36 \%$ and $175 \mathrm{~d}$, respectively (Table 2).

$\boldsymbol{S C C}$. No difference existed in LSCC after enrollment between treatment programs. The model estimate of treatment effect difference using positive control as reference $\left(\right.$ Diff $\left._{\mathrm{PC}}\right), 95 \%$ confidence interval, and $P$ value were $0.1,-0.2$ to 0.5 , and $P=0.443$, respectively (Table 1 and Figure 3 ). The LSCC was numerically higher in cases assigned to positive-control treatment in 3 herds, the same in 2 herds, and lower in 3 of the 8 herds enrolled in the study. No other covariates in addition to the explanatory variable of interest, treatment program, remained in the model because of confounding the treatment program effect on LSCC after enrollment. The autoregressive structure, in which correlations between adjacent repeated SCC measurements are higher than between measurements further apart, resulted in the best model fit, based on various goodness-of-fit measures.

The estimated LSCC for the rest of the lactation after enrollment was 4.2 and 4.4 for cows assigned to positive-control and to culture-based treatment, respectively. The overall LSCC after enrollment for both clinical mastitis treatment programs for no growth, gram-negative, and gram-positive cases were 3.9, 4.4, and 4.8 , respectively (Table 3 ).

Milk Production. No significant difference existed in milk production after enrollment between treatment programs $\left[\operatorname{Diff}_{\mathrm{PC}}(95 \% \mathrm{CI})=0.7(-0.9,2.3) ; P=0.414\right.$; Table 1 and Figure 4]. Milk production was numerically higher in cases assigned to positive-control treatment in 4 herds and lower in 4 of the 8 herds enrolled in the study. No other covariates in addition to the explanatory variable of interest, treatment program, remained in the model because of confounding the treatment program effect on milk production after enrollment. The autoregressive structure also resulted in the best model fit, based on various goodness-of-fit measures.

The estimated daily milk production after enrollment was 30.0 and $30.7 \mathrm{~kg}$ for cows assigned to positive-con- 
Table 1. Treatment effects, model fit statistics, and covariance parameters for random and fixed effects of models evaluating different outcomes ${ }^{1}$

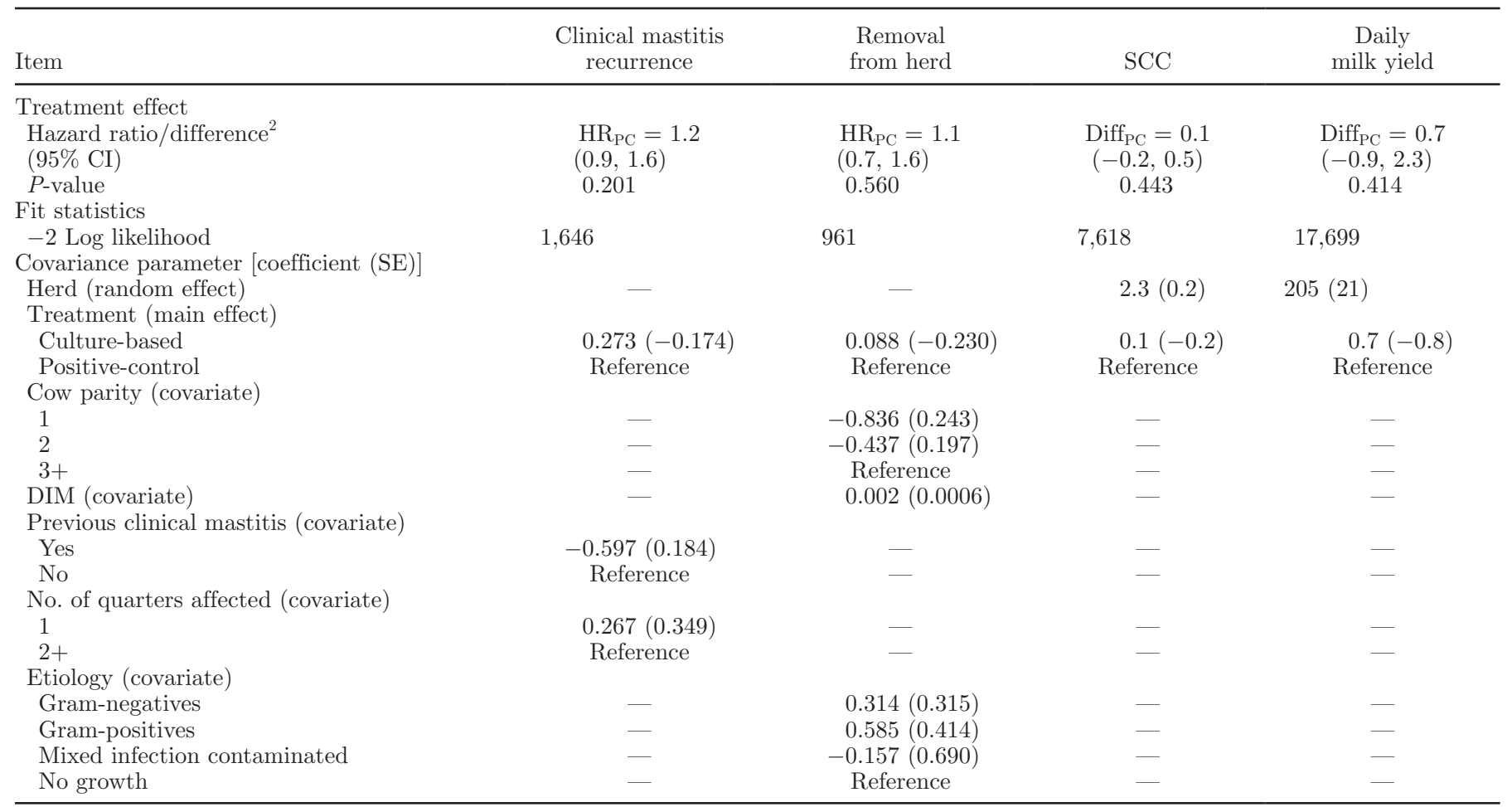

${ }^{1}$ Covariates evaluated for potential confounding included cow parity, DIM at the clinical mastitis event, previous occurrence of a clinical mastitis case in the same quarter in the present lactation, number of quarters affected, case severity, and etiology of infection. Other covariates, such as SCC in the test before the clinical mastitis event, were evaluated only when the estimated outcome was SCC, and milk production in the test before the clinical mastitis event when the estimated outcome was milk production. Severity, SCC, and milk production were not confounders for any of the outcomes evaluated.

${ }^{2} \mathrm{HR}_{\mathrm{PC}}=$ model hazard ratio estimate of treatment effect using positive control as reference; Diff $\mathrm{PC}_{\mathrm{PC}}=$ model estimate of treatment effect difference using positive control as reference.

trol and to culture-based treatment, respectively. The overall daily milk production after enrollment for both clinical mastitis treatment programs for no growth, gram-negative, and gram-positive cases were 31.8, 30.6, and $28.8 \mathrm{~kg}$, respectively (Table 3 ).

\section{Power Analysis}

The current study sample size provided an excess of $95 \%$ confidence and $80 \%$ power to detect a difference in (1) clinical mastitis recurrence risk of $14 \%$ or greater, (2) culling or death risk of $14 \%$ or greater, (3) linear SCC of 0.03 or more, and (4) daily milk yield of $0.2 \mathrm{~kg}$ or more.

\section{DISCUSSION}

Risk for recurrence of a clinical mastitis event in the same quarter was not different between the 2 clinical mastitis treatment programs. Similarly, in a clinical trial, when comparing the clinical mastitis recurrence risk between antibacterial and no antibacterial clinical mastitis treatments, it did not differ for mild clinical mastitis cases where no bacteria or coliforms were isolated (Van Eenennaam et al., 1995). However, in the present study, the recurrence risk of a clinical mastitis event for both clinical mastitis treatment programs was higher than previously reported. It may be due to different infection pathophysiology characteristics or merely due to differences in the definition, detection, and record keeping of clinical mastitis cases among studies. Differences in etiology appeared not to explain the high recurrence risk, as the risk and days to recurrence of a clinical mastitis event was not different among the different clinical mastitis etiology classification groups.

Milk production and LSCC after enrollment was not different between clinical mastitis treatment programs. These results agreed with 2 previous clinical trials for which milk production did not differ between antibacterial and no antibacterial clinical mastitis treatment groups for mild clinical mastitis cases where no bacteria or coliforms were isolated (Van Eenennaam et al., 1995; Roberson et al., 2004). However, another study reported that after resolution of clinical mastitis, cows 
Table 2. Clinical mastitis recurrence and removals from herd for 2 clinical mastitis treatment programs

\begin{tabular}{|c|c|c|c|c|}
\hline \multirow[b]{2}{*}{ Etiology } & \multicolumn{2}{|c|}{ Recurrence of clinical mastitis $\left[\%^{1}(\mathrm{n})^{2}\right]$} & \multicolumn{2}{|c|}{$\begin{array}{l}\text { Removed from herd by culling } \\
\text { or death }[\%(\mathrm{n})]\end{array}$} \\
\hline & Positive-control & Culture-based & Positive-control & Culture-based \\
\hline No growth & $34(68)$ & $48(73)$ & $22(58)$ & $26(65)$ \\
\hline Gram-negatives & $39(56)$ & $52(42)$ & $30(50)$ & $25(36)$ \\
\hline Gram-positives & $29(68)$ & $34(79)$ & $37(49)$ & $35(62)$ \\
\hline All cases & $35(220)$ & $43(210)$ & $28(195)$ & $32(195)$ \\
\hline Herd range $^{3}$ & $23-40$ & $22-63$ & $19-60$ & $11-43$ \\
\hline
\end{tabular}

given antibiotics along with supportive treatment returned to normal performance, whereas cows given only supportive treatment incurred continued loss (Shim et al., 2004). However, the latter study did not evaluate the effect of treatment on milk production depending on the etiology of the clinical mastitis case. In addition, the authors hypothesized that the continued milk yield loss in clinical mastitis cases that did not receive antibiotic therapy could be the result of more persistent subclinical infections or more marked alteration of mammary gland function. That was not the case in this study, as no differences existed between treatment programs in the prevalence of IMI 14 and $21 \mathrm{~d}$ after the clinical mastitis event (Lago et al., 2011).

The risk and days to culling between clinical mastitis treatment programs was not different. These results agree with an earlier clinical trial for which the time to removal from the herd after clinical mastitis did not differ between antibacterial and no antibacterial treatment groups or by etiology of infection (Van Eenennaam et al., 1995).

It has been reported in an accompanying manuscript that the use of an on-farm culture system to guide the strategic treatment of clinical mastitis decreased intra-

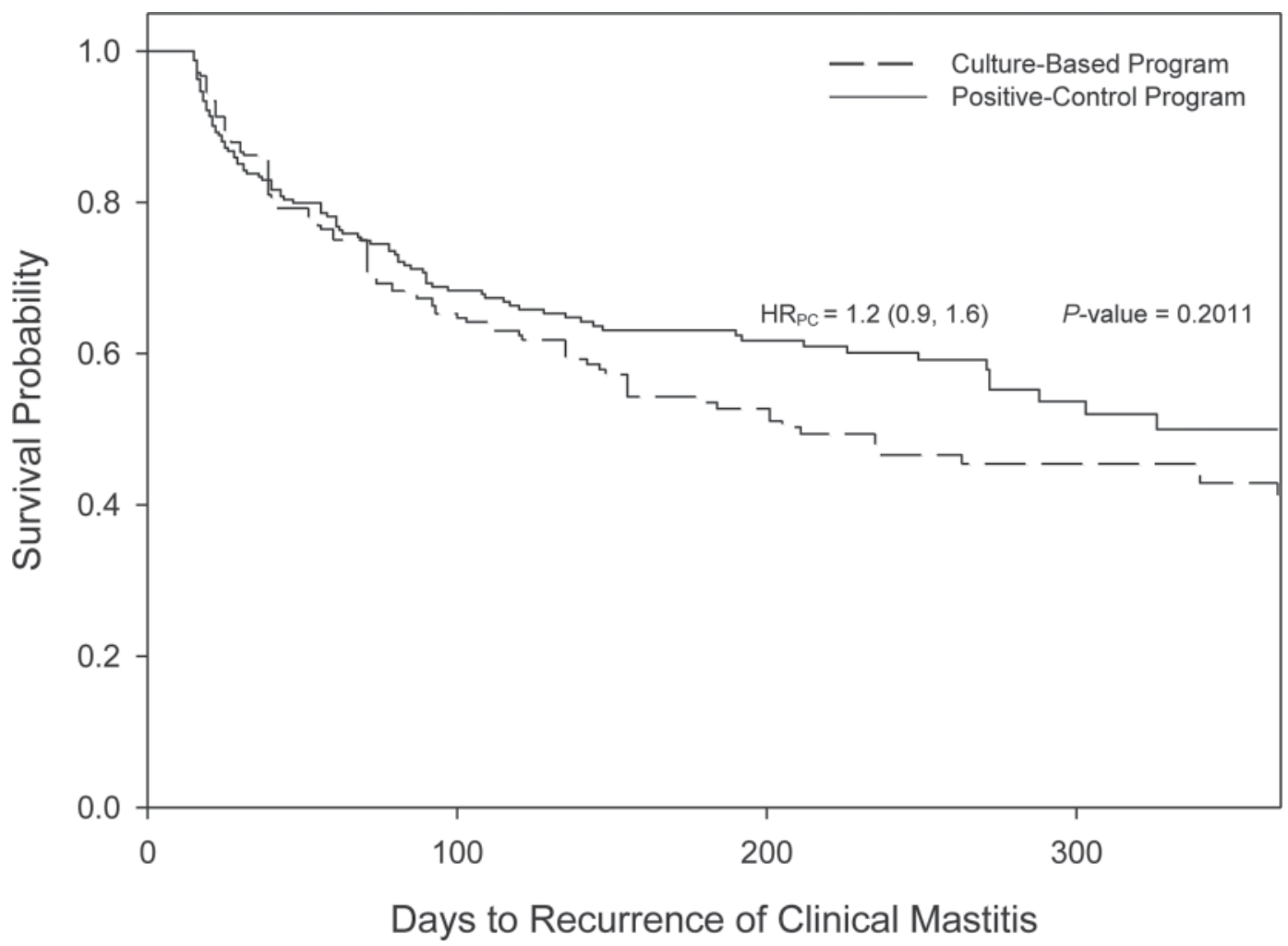

Figure 1. Kaplan-Meier survival graph representing the probability of a recurrence of a clinical mastitis case in the same quarter at a given day after the clinical mastitis event for 2 clinical mastitis treatment programs. $\mathrm{HR}_{\mathrm{PC}}=$ positive-control hazard ratio. 


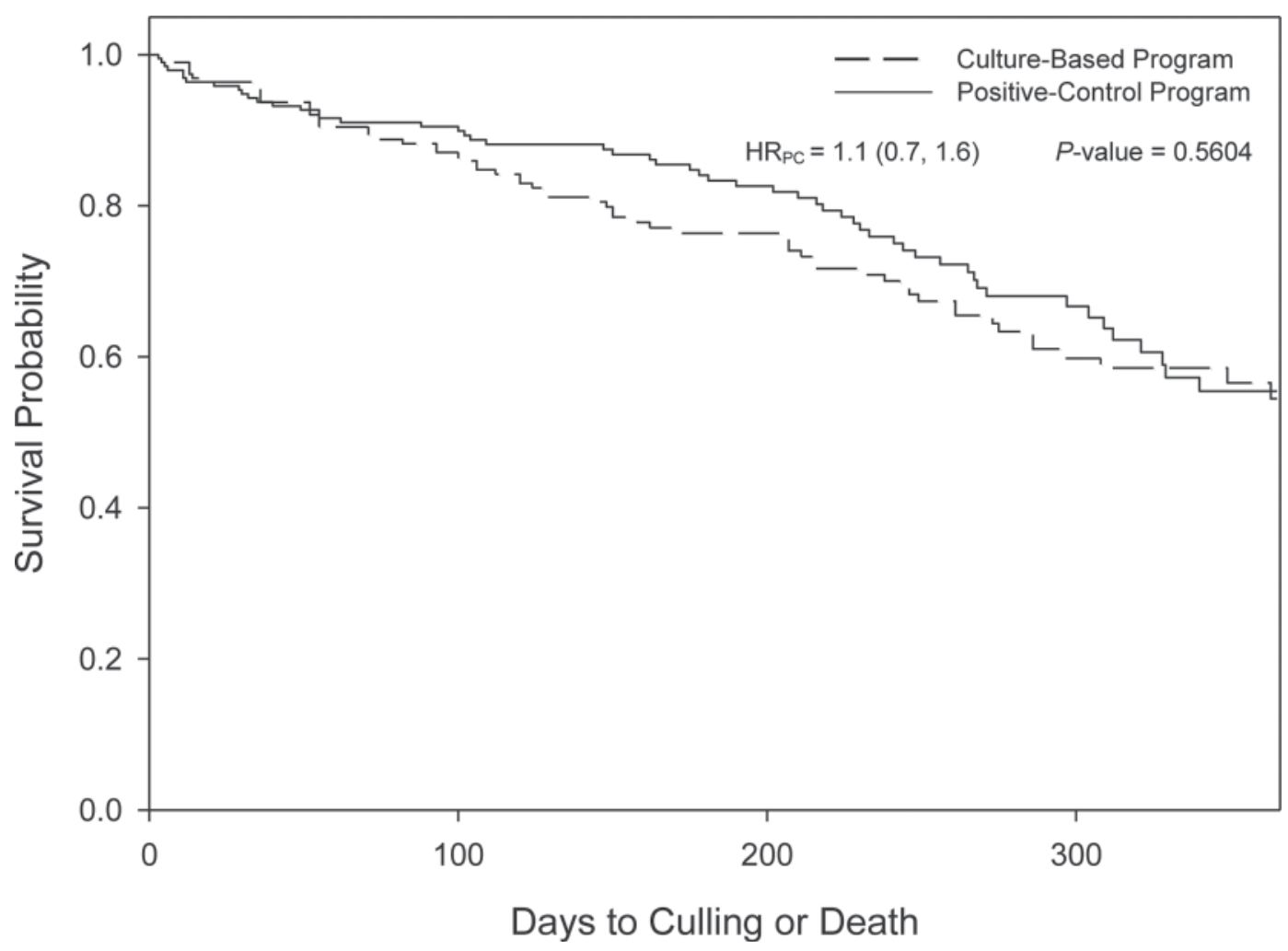

Figure 2. Kaplan-Meier survival graph representing the probability of culling or death at a given day after the clinical mastitis event for 2 clinical mastitis treatment programs. $\mathrm{HR}_{\mathrm{PC}}=$ positive-control hazard ratio.

mammary antibiotic use by half and tended to decrease by 1 day the milk withholding time, without significant differences in short-term clinical and bacteriological outcomes after clinical mastitis (Lago et al., 2011). Additionally, long-term outcomes of the intervention were evaluated in the current manuscript, as they may represent an important component of the overall economic impact of the intervention. The recurrence risk of clinical mastitis appears to be a sensitive indicator of differences in treatment efficacy between treatments. For example, in previous trials, the recurrence risk of clinical mastitis did differ for antibacterial and no antibacterial clinical mastitis treatment groups, in mild clinical mastitis cases where streptococci were isolated (Van Eenennaam et al., 1995). Similarly, several studies reported that after clinical mastitis, quarter milk SCC is higher (Sheldrake et al., 1983; Schepers et al., 1997; de Haas et al., 2002), milk production is lower (Houben et al., 1993; Rajala-Schultz et al., 1999; Wilson et al., 2004), and the risk of culling is higher (Dohoo and Martin, 1984; Erb et al., 1985; Milian-Suazo et al., 1989; Gröhn et al., 1997, 2005; Rajala-Schultz and Gröhn, 1999). Consequently, these outcomes must be evaluated to compare the overall biologic and economic impact of treatment interventions for clinical mastitis.
The strengths of the present study are related to the validation of a program to treat clinical mastitis. The success of the culture-based treatment program not only depends on the inefficacy of antibiotic intramammary treatment in no growth or gram-negative cases, but also depends on the accuracy of the on-farm culture system, and the effects of a 1-d delay to initiate intramammary antibiotic therapy in those quarters selected for treatment. However, it is unknown if the validity of the present study results hold when using antibiotics different than cephapirin sodium.

\section{CONCLUSIONS}

The selective treatment of clinical mastitis based on on-farm culture resulted in no differences in long-term outcomes, such as recurrence of clinical mastitis in the same quarter, SCC, milk production, and cow survival for the rest of the lactation after clinical mastitis. It has been reported in a companion paper that the selective treatment of clinical mastitis based on on-farm culture results halves antibiotic use and tends to decrease milk withholding time without affecting short-term clinical and bacteriological outcomes (Lago et al., 2011). Results of both analyses will be used to evaluate the 
Table 3. Somatic cell count (cells/mL) and daily milk yield $(\mathrm{kg} / \mathrm{d})$ for 2 clinical mastitis treatment programs

\begin{tabular}{lccccc}
\hline & \multicolumn{2}{c}{ Linear SCC $\left[\right.$ mean $\left.\pm \mathrm{SD}(\mathrm{n})^{1}\right]$} & & \multicolumn{2}{c}{ Daily milk yield $[$ mean \pm SD $(\mathrm{n})]$} \\
\cline { 2 - 3 } \cline { 5 - 6 } Etiology & Positive-control & Culture-based & & Positive-control & Culture-based \\
\hline No growth & $3.9 \pm 0.2(57)$ & $4.0 \pm 0.2(60)$ & & $32.2 \pm 1.0(57)$ & $31.4 \pm 1.0(60)$ \\
Gram-negatives & $4.3 \pm 0.3(48)$ & $4.6 \pm 0.3(44)$ & & $30.0 \pm 1.1(48)$ & $31.5 \pm 1.3(44)$ \\
Gram-positives & $4.7 \pm 0.3(52)$ & $4.9 \pm 0.2(63)$ & & $29.1 \pm 1.1(52)$ & $28.6 \pm 1.1(63)$ \\
All cases & $4.2 \pm 0.1(178)$ & $4.4 \pm 0.1(178)$ & & $30.0 \pm 0.6(178)$ & $30.7 \pm 0.6(178)$ \\
Herd range $^{2}$ & $3.2-5.8$ & $3.4-5.4$ & & $26-33$ & $27-39$ \\
\hline
\end{tabular}

${ }^{1} \mathrm{n}$ is the population from which means and standard deviations were calculated.

${ }^{2}$ Herd range for the different outcomes [minimum herd (\%)-maximum herd (\%)].

overall cost-benefit of using an on-farm culture system to guide strategic treatment decisions in cows with mild and moderate clinical mastitis.

\section{ACKNOWLEDGMENTS}

The authors thank Daniel Hagman, Grant Williams, Joseph Hochhalter, Krista Steffenhagen, Mackenzie Jones, Margaret Perala, Maya Kuratomi, and Megan Becker for the project implementation work and the Udder Health Laboratory Staff at the University of Minnesota, College of Veterinary Medicine; Danielle Davignon, Carlo Spanu, Dhananjay Apparao, José
Pantoja, Leane Oliveira, and Martín Pol for the project implementation work and Carol Hulland for the laboratory work at the University of Wisconsin, Dairy Science Department; and Amy Stanton, Cindy Todd, Erin Vernooy, and Nicole Perkins for the project implementation work and Anna Bashiri for the laboratory work at the University of Guelph, Ontario Veterinary College (Canada). We also recognize the input provided by John Fetrow (University of Minnesota, College of Veterinary Medicine, Saint Paul), Michael Oakes (University of Minnesota, School of Public Health, Minneapolis), Paul Rapnicki(University of Minnesota, College of Veterinary Medicine), and Steven Stewart (Univer-

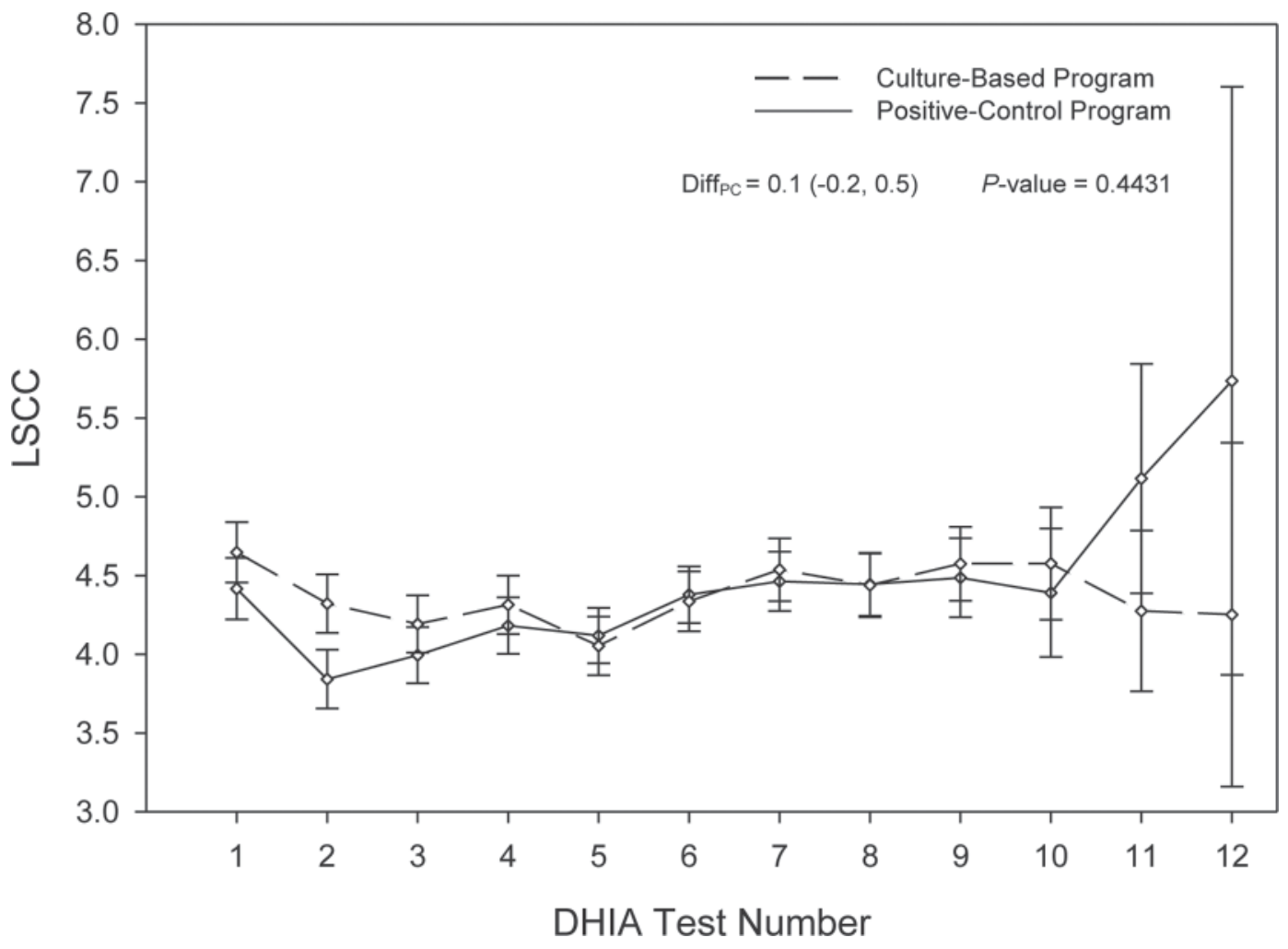

Figure 3. Least square linear SCC (LSCC) mean up to 12 DHIA tests after the clinical mastitis event for 2 clinical mastitis treatment programs. Diff $\mathrm{PC}_{\mathrm{PC}}=$ model estimate of treatment effect difference using positive control as reference. 


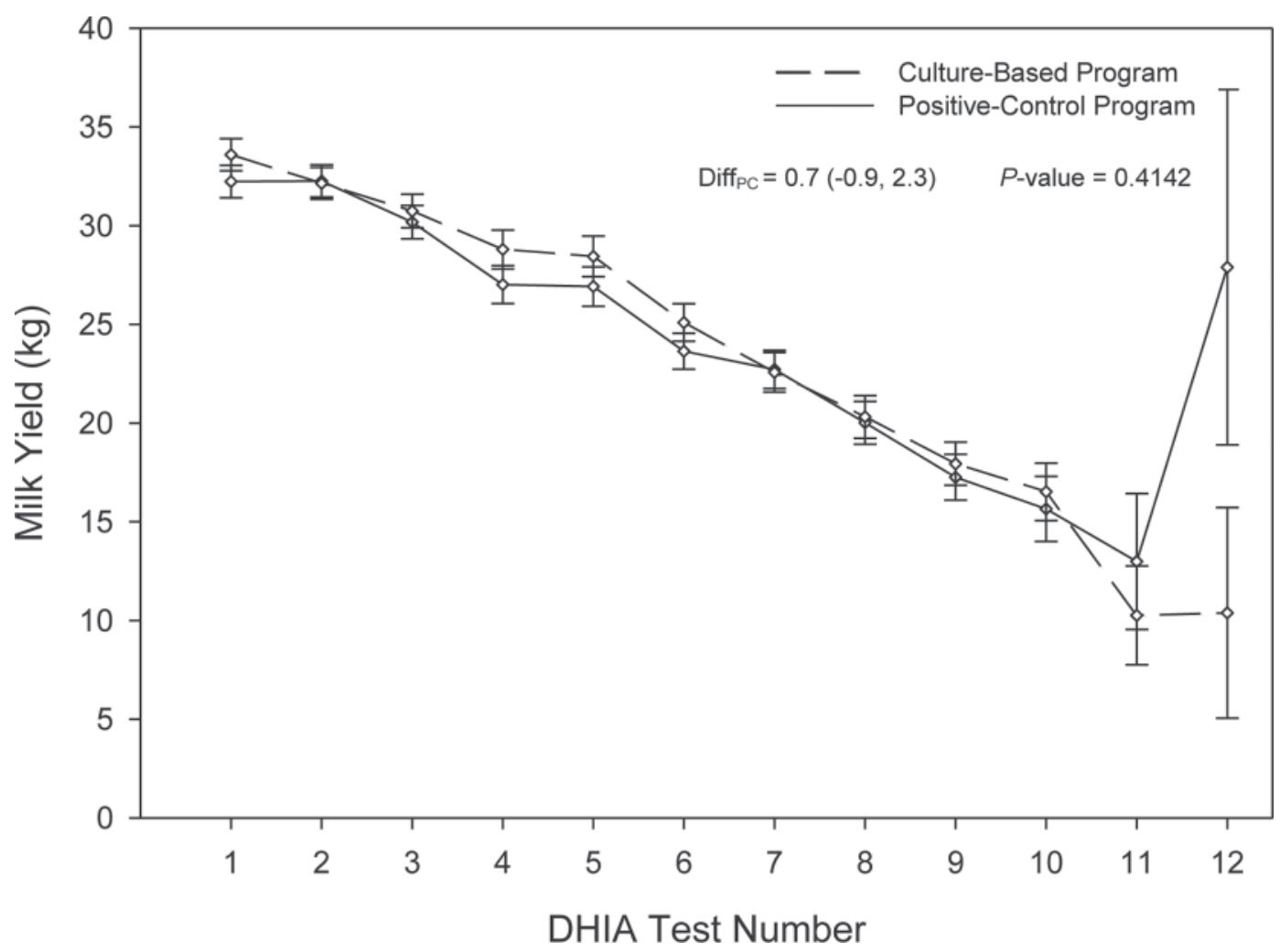

Figure 4. Least square milk yield mean up to 12 DHIA tests after the clinical mastitis event for 2 clinical mastitis treatment programs. Diff ${ }_{\mathrm{PC}}$ $=$ model estimate of treatment effect difference using positive control as reference.

sity of Minnesota, College of Veterinary Medicine). A special thanks to the dairy owners and farm personnel for providing their herds and labor for this project. The study was possible thanks to funding provided by the Minnesota Agriculture Experimental Station, Fort Dodge Animal Health Inc. (Fort Dodge, IA) and Dairy Farmers of Ontario. In particular, the authors remember Dr. Jon Rosenberg, of Fort Dodge Animal Health Inc., who was especially supportive of this study.

\section{REFERENCES}

Ali, A. K. A., and G. E. Shook. 1980. An optimum transformation of somatic cell concentration in milk. J. Dairy Sci. 63:487-490.

Beatty, B., R. Farnsworth, A. Lund, R. Lyon, and G. Ward. 1985. Medium to culture and differentiate coagulase-positive and -negative staphylococci from bovine milk. J. Food Prot. 48:1019-1021.

de Haas, Y., H. W. Barkema, and R. F. Veerkamp. 2002. The effect of pathogen-specific clinical mastitis on the lactation curve of somatic cell count. J. Dairy Sci. 85:1314-1323.

Dohoo, I. R., and S. W. Martin. 1984. Disease, production and culling in Holstein-Friesian cows. V. Survivorship. Prev. Vet. Med. $2: 771-784$.

Erb, H. N., R. D. Smith, P. A. Oltenacu, C. L. Guard, R. B. Hillman, P. A. Powers, M. C. Smith, and M. E. White. 1985. Path model of reproductive disorders and performance, milk fever, mastitis, milk yield, and culling in Holstein cows. J. Dairy Sci. 68:3337-3349.

Erskine, R. J., R. C. Wilson, M. G. Riddell, J. W. Tyler, H. J. Spears, and B. S. Davis. 1992. Intramammary administration of gentami- cin as treatment for experimentally induced Escherichia coli mastitis in cows. Am. J. Vet. Res. 53:375-381.

Gröhn, Y. T., V. Ducrocq, and J. A. Hertl. 1997. Modeling the effect of a disease on risk of culling: An illustration of the use of time-dependent covariates for survival analysis. J. Dairy Sci. 80:1755-1766.

Gröhn, Y. T., R. N. González, D. J. Wilson, J. A. Hertl, G. Bennett, H. Schulte, and Y. H. Schukken. 2005. Effect of pathogen-specific clinical mastitis on herd life in two New York State dairy herds. Prev. Vet. Med. 71:105-125.

Houben, E. H. P., A. A. Dijkhuizen, J. A. M. Arendonk, and R. B. M. Huirne. 1993. Short- and long-term production losses and repeatability of clinical mastitis in dairy cattle. J. Dairy Sci. 76:25612578 .

Lago, A., S. M. Godden, R. Bey, P. L. Ruegg, and K. Leslie. 2011. The selective treatment of clinical mastitis based on on-farm culture results: I. Effects on antibiotic use, milk withholding time, and short-term clinical and bacteriological outcomes. J. Dairy Sci. 94:4441-4456

Milian-Suazo, F., H. N. Erb, and R. D. Smith. 1989. Risk factors for reason-specific culling of dairy cows. Prev. Vet. Med. 7:19-29.

Mitchell, J. M., M. W. Griffiths, S. A. McEwen, W. B. McNab, and A. J. Yee. 1998. Antimicrobial drug residues in milk and meat: Causes, concerns, prevalence, regulations, tests, and test performance. J. Food Prot. 61:742-756.

NMC. 1999. Laboratory Handbook on Bovine Mastitis. National Mastitis Council, Madison, WI

Pol, M., and P. L. Ruegg. 2007. Relationship between antimicrobial drug usage and antimicrobial susceptibility of gram-positive mastitis pathogens. J. Dairy Sci. 90:262-273.

Pyörälä, S., L. Kaartinen, H. Käck, and V. Rainio. 1994. Efficacy of two therapy regimens for treatment of experimentally induced Escherichia coli mastitis in cows. J. Dairy Sci. 77:453-461. 
Rajala-Schultz, P. J., and Y. T. Gröhn. 1999. Culling of dairy cows. Part I. Effects of diseases on culling in Finnish Ayrshire cows. Prev. Vet. Med. 41:195-208.

Rajala-Schultz, P. J., Y. T. Gröhn, C. E. McCulloch, and C. L. Guard. 1999. Effects of clinical mastitis on milk yield in dairy cows. J. Dairy Sci. 82:1213-1220.

Roberson, J. R. 2003. Establishing treatment protocols for clinical mastitis. Vet. Clin. North Am. Food Anim. Pract. 19:223-234.

Roberson, J. R., L. D. Warnick, and G. Moore. 2004. Mild to moderate clinical mastitis: Efficacy of intramammary amoxicillin, frequent milk-out, a combined intramammary amoxicillin, and frequent milk-out treatment versus no treatment. J. Dairy Sci. 87:583-592.

SAS Institute. 2003. User's Guide: Statistics. Version 9.1. SAS Inst. Inc., Cary, NC.

Schepers, A. J., T. J. G. M. Lam, Y. H. Schukken, J. B. M. Wilmink, and W. J. A. Hanekamp. 1997. Estimation of variance components for somatic cell counts to determine thresholds for uninfected quarters. J. Dairy Sci. 80:1833-1840.

Sheldrake, R. F., R. J. T. Hoare, and G. D. McGregor. 1983. Lactation stage, parity, and infection affecting somatic cells, electrical conductivity, and serum albumin in milk. J. Dairy Sci. 66:542-547.
Shim, E. H., R. D. Shanks, and D. E. Morin. 2004. Milk loss and treatment costs associated with two treatment protocols for clinical mastitis in dairy cows. J. Dairy Sci. 87:2702-2708.

Sundlof, S. F., J. B. Kaneene, and R. Miller. 1995. National survey on veterinarian-initiated drug use in lactating dairy cows. J. Am. Vet. Med. Assoc. 207:347-352.

University of Minnesota Laboratory for Udder Health. 2004. Minnesota Easy Culture System II user's manual. University of Minnesota, Saint Paul, MN.

Van Eenennaam, A. L., I. A. Gardner, J. Holmes, L. Perani, R. J. Anderson, J. S. Cullor, and W. M. Guterbock. 1995. Financial analysis of alternative treatments for clinical mastitis associated with environmental pathogens. J. Dairy Sci. 78:2086-2095.

Wilson, D. J., R. N. Gonzalez, J. Hertl, H. F. Schulte, G. J. Bennett, Y. H. Schukken, and Y. T. Gröhn. 2004. Effect of clinical mastitis on the lactation curve: A mixed model estimation using daily milk weights. J. Dairy Sci. 87:2073-2084. 